\title{
VLBI study of the jets in radio-loud narrow-line Seyfert 1 galaxies
}

\section{Minfeng Gu*}

Key Laboratory for Research in Galaxies and Cosmology, Shanghai Astronomical Observatory, Chinese Academy of Sciences, 80 Nandan Road, Shanghai, 200030, China

E-mail: gumfeshao.ac.cn

\begin{abstract}
With relatively small black hole masses and high accretion rates, narrow-line Seyfert 1 galaxies are thought to be young AGNs. About $7 \%$ of them are radio-loud narrow-line Seyfert 1 galaxies (RLNLS1s). RLNLS1s allow us to re-address some of the key questions regarding the physics of jet formation. As the first step of the systematic study on the jet properties of RLNLS1s, we present the radio structure of fourteen RLNLS1s from VLBA observations at $5 \mathrm{GHz}$ in 2013. Although all these sources are very radio-loud with $R>100$, their jet properties are diverse, in terms of their pc-scale morphology and overall radio spectral shape. The core brightness temperatures of our sources are significantly lower than those of blazars, therefore, the beaming effect is generally not significant, compared to blazars. This implies that the bulk jet speed may likely be low in the RLNLS1s in our sample.
\end{abstract}

Revisiting narrow-line Seyfert 1 galaxies and their place in the Universe - NLS1-2018

9-13 April 2018

Padova Botanical Garden, Italy

\footnotetext{
* Speaker.
} 


\section{Introduction}

Narrow-line Seyfert 1 galaxies (NLS1s) are a subclass of active galactic nuclei (AGNs), with strong permitted optical/UV Fe II emission lines, relatively weak forbidden-line emission (i.e., $[\mathrm{OIII}] 5007 / \mathrm{H} \beta<3$ ), and their broad lines are narrower than those of normal broad-line Seyfert 1 galaxies with $\operatorname{FWHM}(\mathrm{H} \beta)$ less than $2000 \mathrm{~km} \mathrm{~s}^{-1}[30,16]$. NLS1s are often thought to be young and still evolving AGNs with relatively small black hole masses and high accretion rates [22]. NLS1s as a class show a low probability to be radio loud at $\sim 7 \%$, compared to normal broad line AGNs [21, 41]. Interestingly, radio-loud NLS1s (RLNLS1s) are inhomogeneous in their radio properties. As shown in [21], most RLNLS1s in their sample are compact, steep spectrum sources, and hence likely associated with compact steep-spectrum (CSS) radio sources, while only a few NLS1s showed blazar-like behaviour. On the other hand, observational evidence has recently shown that a significant fraction of RLNLS1s at the highest radio-loudness values, does display the characteristics of blazars, including large-amplitude radio flux and spectral variability, compact radio cores, very high variability brightness temperatures, enhanced optical continuum emission, flat X-ray spectra, and blazar-like spectral energy distributions [40]. RLNLS1s are at an opposite end of AGN correlation space than classical radio-loud AGNs [36], in terms of small black hole masses, high accretion rate, and very strong Fe II emission [21]. Therefore, RLNLS1s allow us to re-address some of the key questions regarding the physics of jet formation, for example, the physical conditions under which a jet can be launched.

\section{The jet properties of RLNLS1s}

Relativistic jets were shown to exist in a few RLNLS1s using Very Long Baseline Interferometry (VLBI) observations, based on their high brightness temperatures, and their inverted radio spectra [11], which was later confirmed by the detection of flaring $\gamma$-ray emission [1,2]. The previous work based on VLBA data for a few sources argued that RLNLS1s can be either intrinsically radio loud, or apparently radio loud due to jet beaming effects $[17,12]$. These observational results may be contrary to the well-known paradigm that jets are generally associated with elliptical host galaxies in typical radio-loud AGNs, while there is tentative evidence suggesting that at least a few low-redshift RLNLS1s are hosted by spiral galaxies [42, 3, 26]. Although a number of RLNLS1s have been imaged with VLBI [17, 12, 15, 9, 37, 34], comprehensive large-sample studies of their jet properties on parsec scales have just started [18, 19].

From the high-resolution VLBA images, the brightness temperature of the radio core in the rest frame can be estimated [14], which is often used to constrain the jet Doppler factor in combination with the assumed intrinsic brightness temperature, either as the equipartition brightness temperature of $5 \times 10^{10} \mathrm{~K}$ [33], or the inverse Compton catastrophe brightness temperature of $\sim 10^{12} \mathrm{~K}$ [20]. Moreover, the beaming effect of RLNLS1s can be investigated with comparion to blazars, in which the beaming effect is usually strong. The VLBA core brightness temperatures of blazars typically range between $10^{11}$ and $10^{13} \mathrm{~K}$ with a median value near $10^{12} \mathrm{~K}$, and can even extend up to $5 \times 10^{13} \mathrm{~K}[24,25]$. 


\section{$2.1 \gamma$-ray detected sources}

Seventeen NLS1s have been detected in the $\gamma$-ray band by the Fermi/LAT with high significance (test statistic, TS $>25$ ) hitherto ${ }^{1}$ (PMN J0948+0022, [1]; PKS 1502+036, 1H 0323+342 and PKS 2004-447, [2]; SBS 0846+513, [7]; FBQS J1644+2619, [10]; SDSS J1443+4725 and SDSS J1305+5116, [27]; SDSS J1222+0413, [39]; SDSS J0956+2515, [29]; SDSS J2118-0732, [38]; SDSS J0932+5306, GB6 J0937+5008, SDSS J0958+3224, SDSS J1421+3855, TXS 1518+423, and PMN J2118+0013, [31]). Most of these $\gamma$-ray NLS1s possess a one-sided jet extending from a dominant central core, reminiscent of blazars $[8,37]$. The brightness temperature is usually high, well in excess of the equipartition limit and in several cases above the inverse-Compton limit [35]. The values are comparable to those of blazars, strongly favoring the beaming effect in these gamma-ray objects. So far, the six brightest known NLSy1s in terms of radio flux density have been the subject of regular monitoring with the VLBA at $15 \mathrm{GHz}$ as part of the MOJAVE program, and the apparent superluminal motion has been detected in three objects, $1 \mathrm{H} 0323+342$, SBS 0846+513 and PMN J0948+0022, with $\beta_{\text {app }}=9.1 \mathrm{c}, 6.6 \mathrm{c}$, and 9.7c, respectively [28]. SDSS J1305+5116 and SDSS J1443+4725, two NLS1 galaxies both detected in $\gamma$-ray band with high significance and with radio properties similar to CSS sources ${ }^{2}$ [27], show a core-jet structure, with jet extending to about 30 milliarcsecond in the latter (see Fig. 1, and [18]). The core brightness temperature is $\sim 10^{10.8}$ and $\sim 10^{10.3} \mathrm{~K}$ at $5 \mathrm{GHz}$, in SDSS J1305+5116 and SDSS J1443+4725, respectively. The values are at least one order of magnitude lower than most flat-spectrum $\gamma$-ray NLS1 galaxies, indicating intrinsic differences in Doppler boosting of the jet emission.

\subsection{General RLNLS1s}

In order to understand the jet formation in RLNLS1s, the first systematic investigation on the jet properties based on VLBA observations of 14 sources has been carried out [18]. All fourteen NLS1 galaxies were detected as single unresolved sources within 1 arcmin of the SDSS position in both FIRST and NVSS, indicating compact radio structure at FIRST resolution ( $5 \mathrm{arcsec})$. Although all these sources are very radio-loud with $R>100$, their jet properties are diverse, in terms of their milli-arcsecond (mas) scale (pc scale) morphology and their overall radio spectral shape. From VLBA $5 \mathrm{GHz}$ images, seven sources show a compact core only, while the remaining seven objects have core-jet structure (see example images in Fig. 1). The majority of these sources is compact at VLBI mas scales with all components being within $\sim 5$ mas $(\sim 24-38$ pc depending on source redshift). In combination with archival multi-frequency data, seven sources show flat or inverted radio spectra $\left(\alpha \leq 0.5, f_{v} \propto v^{-\alpha}\right)$, while steep spectra $(\alpha>0.5)$ are revealed in the remaining seven objects.

The core brightness temperature ranges from $10^{8.4}$ to $10^{11.4} \mathrm{~K}$ with a median value of $10^{10.1}$ $\mathrm{K}$. This indicates that the radio emission is from non-thermal jets, confirming that powerful jets can be formed in accretion systems with relatively small black hole masses and high accretion rates $[17,12]$. However, the core brightness temperatures of these sources are significantly lower than those of blazars. Therefore, the beaming effect is generally not significant in these sources,

\footnotetext{
${ }^{1}$ Nine more tentative candidates were mentioned by [13, 23, 29].

${ }^{2}$ The CSS-like NLS1 galaxy, PKS 2004-447, was also detected by Fermi/LAT, however, the optical spectral classification as a NLS1 galaxy continues to remain uncertain.
} 

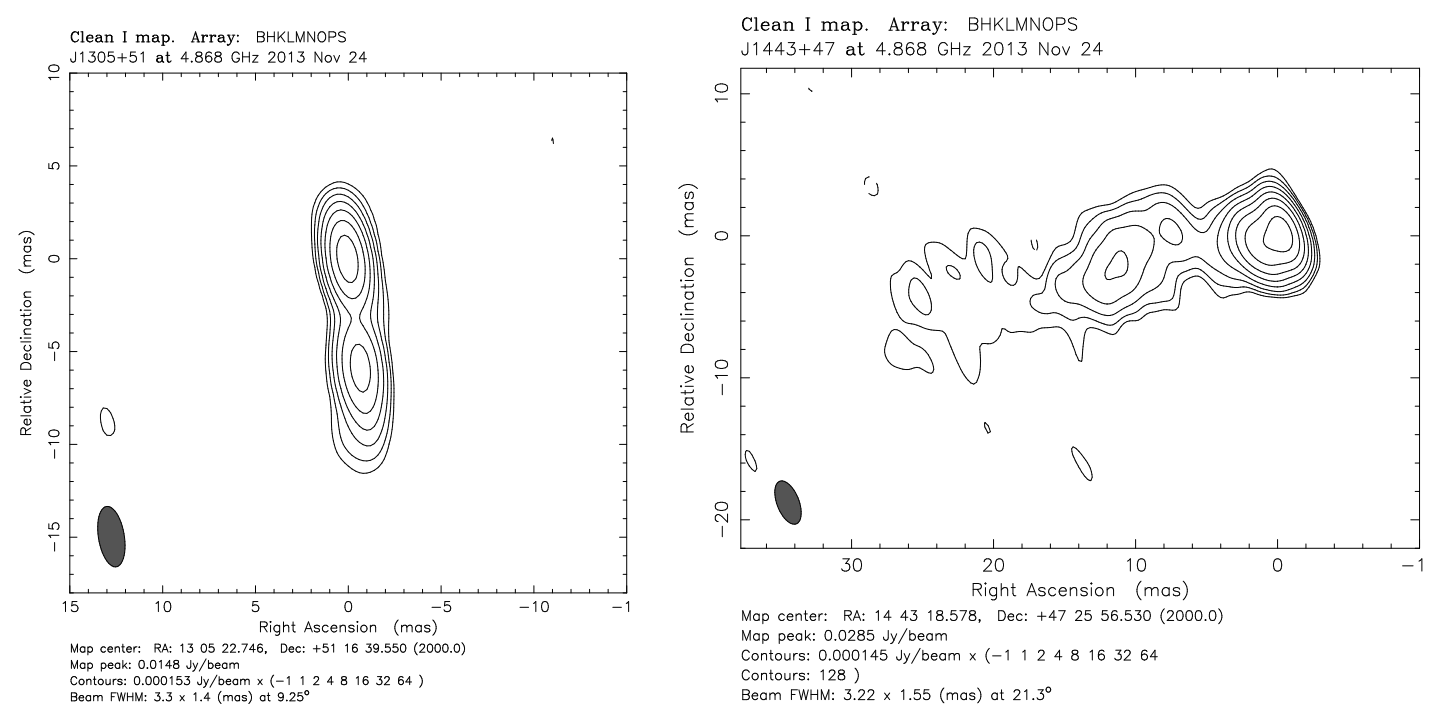

Figure 1: Left: The VLBA 5 GHz image of SDSS J130522.75+511640.3. Right: The VLBA 5 GHz image of SDSS J144318.56+472556.7. Both sources were detected in $\gamma$-ray [27].

implying that the bulk jet speed may likely be low. The evidence for slow jet speeds (i.e., less relativistic jets), in combination with the low kinetic/radio power, may offer an explanation for the compact VLBA radio structure in most sources. The mildly relativistic jets in these high accretion rate systems are consistent with a scenario, where jets are accelerated from the hot corona above the disk by the magnetic field and the radiation force of the accretion disk [5]. Alternatively, a low jet bulk velocity can be explained by low spin in the Blandford-Znajek mechanism [4].

\section{Future work}

To further study the jet properties of RLNLS1s, we will present the VLBA results for a sample of 19 fainter sources in a forthcoming paper (Gu et al., in preparation). The preliminary results are similar with [18], i.e., diverse radio morphology and radio spectra, and lower brightness temperature than blazars. Moreover, in order to directly constrain the jet bulk speed, multi-epoch VLBA observations have been carried out by us to detect the jet proper motion, and source flux variations as well for a sample of ten RLNLS1s. Finally, the multi-frequency polarimetry observations have been done for a newly identified $\gamma$-ray source SDSS J2118-0732 [38] and the CSS-like $\gamma$-ray NLS1 SDSS J1443+4725 [27]. The preliminary results show a core-jet structure in SDSS J2118-0732 and core identification in SDSS J1443+4725, which is uncertain based on the in-band spectral index [18].

From the cross-match between SDSS DR12 and FIRST, the radio emission has been detected in 555 sources out of the overall sample of 11101 NLS1s, in which 378 objects are radio-loud [32]. In addition, 23 radio-detected NLS1s were found in a sample of 167 NLS1s at southern hemisphere, of which ten sources are radio-loud [6]. These samples significantly increase the source number of RLNLS1s, as compared to the sample of 117 RLNLS1s asembled in [18]. Further VLBI studies on more RLNLS1s are needed to explore the jet properties, since the VLBI obervations were only 
performed in several tens of sources. It will also be important to investigate the mechanism of radio emission in radio-quiet NLS1s with VLBI (e.g., star formation and/or jets), for instance, a brightness temperature higher than $\sim 10^{6} \mathrm{~K}$ indicating a most likely jet origin [28].

\section{Acknowledgements}

This work is supported by the National Science Foundation of China (grants 11473054 and U1531245). This conference has been organized with the support of the Department of Physics and Astronomy "Galileo Galilei", the University of Padova, the National Institute of Astrophysics INAF, the Padova Planetarium, and the RadioNet consortium. RadioNet has received funding from the European Union's Horizon 2020 research and innovation programme under grant agreement No 730562.

\section{References}

[1] Abdo A. A. et al. 2009a, ApJ, 699, 976

[2] Abdo A. A. et al. 2009b, ApJ, 707, L142

[3] Antón, S., Browne, I. W. A., \& Marchã, M. J. 2008, A\&A, 490, 583

[4] Blandford, R. D., \& Znajek, R. L. 1977, MNRAS, 179, 433

[5] Cao, X. 2014, ApJ, 783, 51

[6] Chen, S., Berton, M., La Mura, G., et al. 2018, arXiv:1801.07234

[7] D’Ammando, F., Orienti, M., Finke, J., et al. 2012, MNRAS, 426, 317

[8] D’Ammando, F., Orienti, M., Doi, A., et al. 2013a, MNRAS, 433, 952

[9] D’Ammando, F., Orienti, M., Finke, J., et al. 2013b, MNRAS, 436, 191

[10] D'Ammando, F., Orienti, M., Larsson, J., \& Giroletti, M. 2015, MNRAS, 452, 520

[11] Doi A. et al. 2007, PASJ, 59, 703

[12] Doi, A., Asada, K., \& Nagai, H. 2011, ApJ, 738, 126

[13] Foschini, L. 2011, Narrow-Line Seyfert 1 Galaxies and their Place in the Universe, 24

[14] Ghisellini, G., Padovani, P., Celotti, A., \& Maraschi, L. 1993, ApJ, 407, 65

[15] Giroletti, M., Paragi, Z., Bignall, H., et al. 2011, A\&A, 528, L11

[16] Goodrich R.W. 1989, ApJ, 342, 22

[17] Gu, M., \& Chen, Y. 2010, AJ, 139, 2612

[18] Gu, M., Chen, Y., Komossa, S., et al. 2015, ApJS, 221, 3

[19] Gu, M., Chen, Y., Komossa, S., Yuan, W., \& Shen, Z. 2016, Astronomische Nachrichten, 337, 125

[20] Kellermann K. I., Pauliny-Toth I. I. K. 1969, ApJ, 155, L71

[21] Komossa S., Voges W., Xu D., Mathur S., Adorf H. M., Lemson G., Duschl W., Grupe D. 2006, AJ, 132,531

[22] Komossa S. 2008, Rev. Mex. AA Ser. Conf., 32, 86 
[23] Komossa, S., Xu, D., Fuhrmann, L., et al. 2015, A\&A, 574, A121

[24] Kovalev, Y. Y., Kellermann, K. I., Lister, M. L., et al. 2005, AJ, 130, 2473

[25] Kovalev, Y. Y., Aller, H. D., Aller, M. F., et al. 2009, ApJL, 696, L17

[26] León Tavares, J., Kotilainen, J., Chavushyan, V., et al. 2014, ApJ, 795, 58

[27] Liao, N.-H., Liang, Y.-F., Weng, S.-S., Gu, M.-F., \& Fan, Y.-Z. 2015, arXiv:1510.05584

[28] Lister, M. L., 2018, in this proceedings, arXiv:1805.05258

[29] Miller, H., Maune, J., Eggen, J., et al. 2017, Galaxies, 5, 20

[30] Osterbrock D. E., \& Pogge R. W. 1985, ApJ, 297, 166

[31] Paliya, V. S., Ajello, M., Rakshit, S., et al. 2018, ApJL, 853, L2

[32] Rakshit, S., Stalin, C. S., Chand, H., \& Zhang, X.-G. 2017, ApJS, 229, 39

[33] Readhead A.C.S. 1994, ApJ, 426, 51

[34] Richards, J. L., \& Lister, M. L. 2015, ApJL, 800, L8

[35] Schulz, R., Kreikenbohm, A., Kadler, M., et al. 2016, A\&A, 588, A146

[36] Sulentic, J. W., Zamfir, S., Marziani, P., \& Dultzin, D. 2008, Revista Mexicana de Astronomia y Astrofisica Conference Series, 32, 51

[37] Wajima, K., Fujisawa, K., Hayashida, M., et al. 2014, ApJ, 781, 75

[38] Yang, H., Yuan, W., Yao, S., et al. 2018, MNRAS, 477, 5127

[39] Yao, S., Yuan, W., Komossa, S., et al. 2015, AJ, 150, 23

[40] Yuan W., Zhou H. Y., Komossa S., Dong X. B., Wang T. G., Lu H. L., Bai J. M. 2008, ApJ, 685, 801

[41] Zhou H., Wang T., Yuan W., Lu H., Dong X., Wang J., Lu Y. 2006, ApJS, 166, 128

[42] Zhou H. et al., 2007, ApJ, 658, L13 